\title{
A Computerized Assessment of Verbal and Visuospatial Memory (Dys)functions in Patients with Rheumatoid Arthritis
}

This article was published in the following Dove Press journal: Psychology Research and Behavior Management

\author{
Cristian Vasile Petra (D) \\ Laura Visu-Petra (iD ${ }^{2}$ \\ Monica Buta iD ${ }^{2}$ \\ Maria Magdalena Tămaș ${ }^{\prime}$ \\ Oana Benga ${ }^{2}$ \\ Simona Rednic ${ }^{\prime}$ \\ 'Department of Rheumatology, "Iuliu \\ Hatieganu" University of Medicine and \\ Pharmacy, Cluj-Napoca, Romania; \\ ${ }^{2}$ Developmental Psychology Lab, \\ Department of Psychology, Babeș-Bolyai \\ University, Cluj-Napoca, Romania
}

Purpose: Rheumatoid arthritis (RA) is a chronic inflammatory systemic disease associated with various degrees of impairment across different cognitive domains. We aimed to provide a detailed computerized investigation of verbal and visuospatial short-term and working memory (dys)functions in RA patients, assessing both accuracy and response speed, while relating them to age, disease-related activity, affective problems, psychomotor speed and other clinical parameters.

Patients and Methods: The study included 29 RA patients (mean age $50.6 \pm 12.3$ years, $79 \%$ female) and 30 controls (matched according to age, gender and education), assessed with short-term and working memory tasks from the Cambridge Neuropsychological Test Automated Battery (CANTAB) and the Automated Working Memory Assessment (AWMA). Results: RA patients were significantly slower on the basic processing speed test (Motor Screening Test, $p=0.003$ ). Their short-term information storage (verbal and visuospatial) was comparable to controls, yet this similar accuracy came at the expense of a longer response time to retain information correctly (on spatial span, $\mathrm{p}=0.04$ ). On tasks with higher executive demands, both visuospatial and verbal working memory were compromised, as RA patients took longer ( $\mathrm{p}$ $=0.004)$ and had a higher number of total errors $(\mathrm{p}=0.02)$ when conducting a strategic memoryguided search (Spatial Working Memory), and had a significantly lower verbal working memory span on the backwards digit recall test $(\mathrm{p}=0.02)$.

Conclusion: The findings of this study emphasize the usefulness of performing computerized tests to detect subtle signs of cognitive impairment and of intact performance, which can inform memory training protocols for this vulnerable population.

Keywords: rheumatoid arthritis, verbal working memory, visuospatial working memory, cognitive dysfunction, neuropsychological assessment

\section{Introduction}

Rheumatoid arthritis (RA) is a chronic inflammatory systemic disease that primarily affects the connective tissue, with various extra-articular manifestations ranging from cardiovascular disease to cognitive impairment. ${ }^{1}$ The reported prevalence of cognitive dysfunction in RA patients varies from $31 \%$ to $71 \%,{ }^{2,3}$ and a history of RA diagnosed in midlife increases the risk of developing cognitive impairment over a period of 21 years. ${ }^{4}$ In the context of a documented discrepancy between subjective and objective indicators of cognitive impairment, ${ }^{5}$ the use of objective neuropsychological measures is recommended and has so far revealed that RA patients underperform on various cognitive measures of visuospatial processing,
Correspondence: Laura Visu-Petra Department of Psychology, Babeș-Bolyai University, Republicii Str. No. 37, Cluj-

Napoca 400015, Romania

Tel +40264590559

Email laurapetra@psychology.ro 
verbal functioning, short-term memory and executive control. ${ }^{6}$ Such cognitive impairments may be attributed to the disease itself, to the associated clinical features (pain, fatigue), ${ }^{7}$ to medication and/or to psychological conditions (depression, anxiety, stress), often more prevalent in this category of patients than in the general population. ${ }^{8}$ More recently, studies using functional magnetic resonance imaging (fMRI) in RA patients while performing cognitive tasks revealed altered brain network activity associated with inflammation and a compensatory activation of brain regions to offset structural changes, pointing to central nervous system involvement in the context of chronic peripheral inflammation. ${ }^{9}$ Although the exact pathogenic pathways of the resulting cognitive impairment in RA are not yet fully understood, a cluster of interconnected affective, cardiovascular, metabolic and medication-related mechanisms have been suggested, all in the context of a compromised blood-brain barrier integrity due to the chronic inflammatory state. ${ }^{10}$

Most of the cognitive assessment tools administered to RA patients are basic cognitive screening tests such as the Mini-Mental State Examination (MMSE), ${ }^{11}$ or the Montreal Cognitive Assessment. ${ }^{12}$ Although useful to assess global impairment or to eliminate associated conditions such as dementia, these instruments lack the sensitivity and objectivity needed to identify more subtle, subclinical signs of impairment.

Traditional neuropsychological assessment batteries offer a more nuanced picture of cognitive impairment for various clinical conditions across distinct functional domains. Using various such approaches, several studies have linked memory deficits to different autoimmune diseases, ${ }^{6}$ with some studies identifying more pronounced impairments either in visuospatial, ${ }^{13}$ or in verbal memory. ${ }^{5}$ A study using multiple paper-pencil and verbal tests of memory functioning in the case of a fibromyalgia revealed that only the more executive demanding, long-term recall measures (especially visuospatial) were affected, while simple short-term memory remained unaffected. ${ }^{14}$ An innovative approach was put forward by Barraclough and colleagues, who combined a functional magnetic resonance (fMRI) approach with tests from the computerized Cambridge Neuropsychological Test Automated Battery (CANTAB) to examine brain responses to working memory tasks in patients with systemic lupus erythematosus. ${ }^{15}$ The study identified a dissociated profile, with deficits in sustained attention, and compensatory brain activity to maintain similar levels of working memory performance when compared to healthy controls. An advantage of such computerized neuropsychological batteries is that they represent sensitive, standardized tools which offer both speed and accuracy indexes of performance. ${ }^{16}$ They allow the researcher to control for baseline differences in processing speed or motor impairments using simple reaction time measures. This can help clarify if the identified cognitive impairments stem from motor difficulties, overall speed deficits or more circumscribed cognitive deficits. ${ }^{16}$

Our study aimed to provide a detailed investigation of spatial versus verbal short-term and working memory similar to the one conducted by Kim et al ${ }^{14}$ using traditional pencil and paper tests for patients with fibromyalgia. Our target was a sample of patients with RA, tested with more sensitive measures from two computerized neuropsychological batteries, $\mathrm{CANTAB}^{17}$ and Automated Working Memory Assessment (AWMA). ${ }^{18}$ CANTAB tasks have shown sensitivity to cognitive dysfunction and have been widely used across different clinical groups, ${ }^{15,19-21}$ yet to our knowledge, they have not been administered to patients with RA. We aimed to provide a detailed computerized investigation of verbal and visuospatial short-term and working memory (dys)functions in RA patients, assessing both accuracy and response speed, while controlling for variables known to influence cognitive function in this condition (age, disease-related activity, affective problems, other clinical parameters).

Our selected simple and complex working memory span tests rely on sustained executive attention and working memory processes, crucial for the completion of many daily activities of RA patients. Their successful execution is largely mediated by frontal lobe functions, with documented interactions between the neuroanatomical pathways which characterize nociceptive and executive functioning systems in this population. ${ }^{7}$ Some studies show unimpaired simple and complex working memory span performance, with deficits in self-paced attention and long-term recall, partially explained by the association with measures of psychomotor speed and coordination. ${ }^{22,23}$ Other studies however do point to simple and complex span deficits (especially visuospatial) in this population. ${ }^{13,24}$ The only study to provide both accuracy and response speed outcomes extracted from a series of memory tests (measuring both short-term and delayed recognition) showed that basic deficits in sensorimotor speed (related to long-term use of immunosuppressive agents) were the key element underlying recognition 
memory deficits. ${ }^{16}$ We wanted to check for the presence of working memory deficits in RA patients using a brief computerized battery focused on working memory tasks that require sustained attention, self-initiated monitoring and short-term recall processes, with a touchscreen or verbal response that minimizes and controls for reliance on psychomotor speed. We anticipated that the more executive demanding working memory tests (strategic memory search, backward span) would be more affected than the short-term memory measures (digit span, spatial span), which only require information storage. This type of investigation can provide rich avenues for a more nuanced approach to the accurate identification of working memory impairments in patients with RA, and for the development of early therapeutic approaches.

\section{Patients and Methods Study Design, Participants and Clinical Evaluation}

This cross-sectional study included 29 RA patients from the Department of Rheumatology of the "Iuliu Hatieganu" University of Medicine and Pharmacy, Cluj-Napoca, Romania with a mean age of $50.6 \pm 12.3$ years, $79 \%$ female. The patients were fulfilling the 2010 American College of Rheumatology (ACR)/European League against Rheumatism (EULAR) classification criteria. ${ }^{25}$ The exclusion criteria consisted of diabetes mellitus, malignancy, infections, diagnosed neurological or psychiatric diseases that could affect cognition, drugs or alcohol abuse. Thirty participants from the outpatient service of the 2nd Internal Medicine Department of the same University, and matched according to age, gender and education, without any known cognitive deficits and in good general health constituted the control group. All participants provided their informed consent prior to enrollment. The study was approved by the Ethics Committee of the "Iuliu Hatieganu" University of Medicine and Pharmacy Cluj-Napoca, Romania and conducted in accordance with the Declaration of Helsinki.

Initially, we conducted a standardized sociodemographic interview and a detailed clinical evaluation. For the RA group, disease activity was assessed using the Disease Activity Score-28 using CRP (DAS28-CRP) ${ }^{26}$ and functional status was evaluated by the Health Assessment Questionnaire (HAQ), ${ }^{27}$ two of the most widely used tools in daily clinical practice.

\section{Laboratory Measurements}

After overnight fasting, blood samples were withdrawn by venipuncture from all participants to measure serum levels of glucose, total cholesterol, high-density lipoprotein cholesterol (HDL-C) and triglycerides, using standard methods (Cobas Mira Plus analyzer, Hoffman La Roche, Switzerland). C-reactive protein (CRP) and IgM rheumatoid factor (RF, negative $<32 \mathrm{UI} / \mathrm{mL}$ ) were determined by automated turbidimetry, and anti-cyclic citrullinated peptide antibodies (anti-CCP, negative $<5 \mathrm{UI} / \mathrm{mL}$ ) by enzymelinked immunosorbent assay (ELISA).

\section{Objective Cognitive Function Assessment}

As an initial screening for cognitive impairment, we administered the MMSE, ${ }^{11}$ with good internal consistency of the Romanian version ${ }^{28}$ (Cronbach's alpha $=0.80$ for the standard version). The test has a maximum score of 30 , with lower scores indicating more severe cognitive problems. The established cut-off point defining "normal" cognitive function is usually set at 24 .

The CANTABeclipse ${ }^{\mathrm{TM}}$ (Cambridge Cognition Limited, United Kingdom) software version 3.0.0 subtests ${ }^{17}$ were administered on another day by trained research assistants in a quiet, temperature-controlled environment, with adequate lighting. Three tests were selected and run on a touchscreen tablet computer taking approximately 25 minutes to administer.

We first administered the Motor Screening Test (MOT), which screens for basic difficulties with vision, movement and comprehension. Participants touched crosses that appeared on the screen and the computer measured the speed and accuracy of their responses. Next, we assessed spatial short-term memory using the clinical mode of the Spatial Span Test (SSP), a computerized version of the Corsi Block task. A pattern of white boxes was shown on the screen and some boxes changed color in a variable sequence. The participant selected the boxes which changed color in the same order that they had been previously displayed. Outcomes included span length, errors, number of attempts and response duration. We evaluated Spatial Working Memory (SWM) with the SWM test. The task assesses the ability to retain and mentally manipulate spatial information. Participants were required to conduct a self-organized strategic search in order to find tokens that were hidden inside boxes displayed on the screen. The rule of the search is that a token cannot be found in a box that 
previously contained another token. Therefore, checking a box in which a token was hidden before was considered an error (between errors) and so was revisiting a box already found to be empty during the same search (within error). The task increased in complexity through the number of boxes that were presented, ranging from three to eight. The output also included a heuristic strategy score, in the case of which, higher values indicated a poorer strategy. An adjusted Inverse Efficiency (IE) score was computed by subtracting the mean speed on MOT from the mean reaction time of the SSP/SWM tests and then dividing the result by the proportion of correct responses to that test. This measure accounted for potential speedaccuracy tradeoffs, while taking into account individual differences in motor response speed. ${ }^{16}$

Verbal short-term and working memory were assessed with the digit recall and backward digit recall tasks from the translated AWMA battery. ${ }^{18,29}$ During these tests, participants were required to repeat in the same (digit recall) or reversed order (backward digit recall) 6 series of $3,4,5$, 6 and 7 digits that were presented to them in a computerized recording. Aggregate scores for shortterm memory spans were computed following the procedure as previously described. ${ }^{29}$ The verbal working memory tasks from the AWMA also achieved an optimal standardization in administrating procedures, although the verbal responses were recorded by an examiner.

\section{Subjective Health and Affective Functioning Measures}

The brief 21-item version of the Depression Anxiety Stress Scales (DASS) ${ }^{30}$ is a self-report measure of negative affect over the course of the past week, developed to differentiate between the affective symptoms of depression, anxiety and tension/stress. The internal consistency of the Romanian version $^{31}$ was acceptable for all scales and comparable to existing research (Cronbach's alpha $=0.88,0.85$, and 0.86 for Depression, Anxiety and Stress subscales, respectively).

\section{Statistical Analysis}

Statistical analysis was performed using SPSS (Version 20 - IBM Corporation, USA). All variables were checked for normality of distribution using the KolmogorovSmirnov test. Next, generalized linear modeling (GzLM) was used to compare RA patients to controls, adjusting for confounds (age, sex, education). Linear or gamma distribution with identity or log link function was selected, depending on the distribution of the outcome. Raw scores of the selected CANTAB tests measures were used for comparisons. Additionally, each individual percentile score ("better than" \% of normative participants) was extracted from the matched internal normative database of CANTAB (healthy volunteers of comparable age and gender). Finally, correlations between the cognitive and the affective or disease-related variables within the RA sample were computed (Spearman's Rho).

\section{Results}

Demographic and clinical characteristics of the RA patients and controls are detailed in Table 1. The two groups did not significantly differ in terms of age, gender, years of schooling, or other commonly investigated clinical parameters.

RA patients did not significantly differ from controls in their MMSE scores $(p=0.96)$.

The cognitive tests from the CANTAB battery revealed a mixed pattern of deficits and intact performances of RA patients when compared to the control group (see Table 2), or to the normative CANTAB population (see Figure 1). RA patients were significantly slower (reaction time) on the basic processing speed test (MOT, p $=0.003$ ). When looking at the visuospatial memory tests, they did not differ from controls on the spatial short-term memory measure (SSP), presenting a similar spatial span $(\mathrm{p}=$ 0.43 , n.s). However, when looking at the adjusted IE score, they took significantly longer to respond correctly to the test items $(\mathrm{p}=0.04)$.

RA patients had a significantly higher number of errors on the strategic working memory test (SWM). This was visible in terms of both between errors $(p=0.02)$ and total errors $(p=0.02)$, but not in terms of overall search strategy ( $p=0.14$, n.s.). The adjusted IE score indicates that RA patients took significantly longer to correctly search for items $(p=0.004)$, even when psychomotor speed differences were taken into account.

On the verbal memory tests from the AWMA battery, RA patients did not differ in their digit recall test $(\mathrm{p}=$ 0.21 , n.s.), yet they showed impairments in their verbal working memory performance on the backward digit recall test $(\mathrm{p}=0.02)$.

Finally, when looking at the measures of affective difficulties from the DASS, RA patients reported a significantly higher level of depressive symptoms ( $p=$ 
Table I Baseline Characteristics of the RA Patients and Control Group

\begin{tabular}{|c|c|c|c|}
\hline Variables & $\begin{array}{l}\text { RA Patients } \\
(n=29)\end{array}$ & $\begin{array}{l}\text { Controls } \\
(n=30)\end{array}$ & p value \\
\hline Age, years & $50.6 \pm 12.3$ & $51.2 \pm 13.8$ & 0.87 \\
\hline Women, n (\%) & $23(79.3)$ & $22(73.3)$ & 0.60 \\
\hline $\begin{array}{l}\text { Education (years of } \\
\text { schooling) }\end{array}$ & $12.6 \pm 3$ & $13.9 \pm 2.8$ & 0.09 \\
\hline BMI, $\mathrm{kg} / \mathrm{m}^{2}$ & $26.9 \pm 5.3$ & $25.2 \pm 4.3$ & 0.18 \\
\hline WC, $\mathrm{cm}$ & $90.7 \pm 13.2$ & $90.4 \pm 12.3$ & 0.93 \\
\hline $\mathrm{TC}, \mathrm{mg} / \mathrm{dL}$ & $205 \pm 44$ & $204.3 \pm 54.6$ & 0.92 \\
\hline HDL-C, mg/dL & $55.5 \pm 13.2$ & $52.3 \pm 16.8$ & $0.4 I$ \\
\hline $\mathrm{TG}, \mathrm{mg} / \mathrm{dL}$ & $117.3 \pm 48.7$ & $123.8 \pm 75.5$ & 0.70 \\
\hline LDL-C, mg/dL & $126 \pm 39.5$ & $125 \pm 54.6$ & 0.94 \\
\hline MetS, n (\%) & $6(20.7)$ & $9(30)$ & 0.55 \\
\hline Dyslipidemia, n (\%) & $14(48.3)$ & $15(50)$ & 0.93 \\
\hline Hypertension, n (\%) & $6(20.7)$ & $5(16.7)$ & 0.75 \\
\hline Current smoking, n (\%) & $4(13.8)$ & $5(16.7)$ & 0.52 \\
\hline \multicolumn{4}{|l|}{ RA features } \\
\hline $\begin{array}{l}\text { Disease duration, } \\
\text { years }\end{array}$ & $9(4-15.5)$ & - & \\
\hline $\mathrm{ESR}, \mathrm{mm} / \mathrm{h}$ & $15(8.5-26.5)$ & - & \\
\hline CRP, mg/L & $5(2-12)$ & - & \\
\hline DAS28-CRP & $3.9 \pm 1.2$ & - & \\
\hline HAQ & $\mathrm{I} \pm 0.6$ & & \\
\hline RF positivity, n (\%) & $21(72.4)$ & - & \\
\hline Anti-CCP positivity, n (\%) & $23(79.3)$ & - & \\
\hline \multicolumn{4}{|l|}{ Antirheumatic agent use } \\
\hline Prednisone $\mathrm{n}(\%)$ & $7(24.1)$ & - & \\
\hline $\begin{array}{l}\text { Synthetic DMARD, } \\
\text { n (\%) }\end{array}$ & $16(55.2)$ & - & \\
\hline $\begin{array}{l}\text { Biologic DMARD, } \\
\mathrm{n}(\%)\end{array}$ & $6(20.7)$ & - & \\
\hline
\end{tabular}

Notes: Values are expressed as mean $\pm \mathrm{SD}$, median [interquartile range] or $\mathrm{n}(\%)$ $\mathrm{p}<0.05$ is statistically significant.

Abbreviations: BMI, body mass index; WC, waist circumference; TC, total cholesterol; HDL-C, high-density lipoprotein cholesterol; TG, triglycerides; LDL-C, low-density lipoprotein cholesterol; MetS, metabolic syndrome; ESR, erythrocyte sedimentation rate; CRP, C-reactive protein; DAS28-CRP, Disease Activity Score 28 Joints Count-CRP; HAQ, Health Assessment Questionnaire; RF, rheumatoid factor; Anti-CCP, anticitrullinated peptide antibody; DMARD, disease-modifying antirheumatic drug.

$0.03)$, yet they did not differ from controls in terms of anxiety $(\mathrm{p}=0.13)$, or of stress $(0.08)$.

Bivariate correlations (Spearman's rho) between cognitive and affective measures and disease-related parameters in the RA group are displayed in Table 3. Age was negatively related to the strategic WM search indexes (SWM errors, strategy and Inverse Efficiency) and to the verbal WM measure (backward digit span). Patients with higher BMI had a lower SSP span and a higher number of errors on the SWM task and took longer to perform the SWM correctly (SWM IE). This inverse measure of SWM efficiency was also related to disease duration. Finally, higher education (years of schooling) was negatively related to all affective problems (depression, anxiety, stress). There was convergence between higher subjective reports of anxiety (and marginally stress) and poorer overall health assessment (HAQ), with greater disease activity (DAS28-CRP) also being related to higher depression.

\section{Discussion}

In the current study, an objective neuropsychological assessment was conducted to investigate memory dysfunctions in patients with RA, while controlling for sociodemographic, affective and sensorimotor characteristics. To our knowledge, this is the first study focusing on memory deficits in patients with RA using a standardized computerized battery with touchscreen technology, while taking into account information modality (verbal versus visuospatial) and processing demands (low for short-term memory versus high for working memory tasks). Results indicate that short-term information storage - both verbal and visuospatial - was comparable to controls in RA patients, yet this comparable accuracy came at the expense of a longer time needed to retain information correctly (in the case of the spatial span). Unfortunately, a comparable response length index was not recorded for the verbal span task, so similar efficiency costs might exist, but they were not objectively assessed.

When RA patients were confronted with higher executive demands, both their visuospatial and verbal working memory was compromised, relative to controls. More specifically, RA patients took longer to conduct a strategic memory-guided search and had a higher number of total errors, especially incorrectly revisiting boxes where treats had already been found (between errors). However, their actual search strategy did not differ from controls, and they did not have more searches through previously empty locations (within errors). Importantly, all the identified differences remained significant even when controlling for variables such as age, sex, education, and depression (and psychomotor speed in the case of SSP and SWM). Also, the differences between the RA group and the control group remained significant even when contrasting them to the normative CANTAB sample, which indicates particular areas of impairment for RA patients in simple reaction time (MOT) and strategic search (SWM). However, the comparison with the 
Table 2 Cognitive and Affective Assessment in Patients with RA Compared to the Control Group

\begin{tabular}{|c|c|c|c|c|c|}
\hline \multirow[t]{2}{*}{ Variables } & \multicolumn{2}{|c|}{ RA Patients $(n=29)$} & \multicolumn{2}{|c|}{ Controls $(n=30)$} & \multirow[t]{2}{*}{ p value } \\
\hline & Mean \pm SD & Range & Mean \pm SD & Range & \\
\hline MMSE & $28.3 \pm 1.3$ & $25-30$ & $28.3 \pm 1.8$ & $25-30$ & 0.96 \\
\hline MOT mean latency (ms) & $1124 \pm 438$ & $711-2478$ & $884 \pm 205$ & $542-1295$ & $0.003 * *$ \\
\hline MOT errors & $10 \pm 2.2$ & $4.6-14$ & $10.3 \pm 2.4$ & $5-16.4$ & 0.57 \\
\hline SSP span length & $5 \pm 1.5$ & $2-8$ & $5 \pm 1.2$ & $3-7$ & 0.43 \\
\hline SSP total errors & $11.8 \pm 5.7$ & $5-28$ & $10.1 \pm 2.9$ & $5-17$ & 0.53 \\
\hline SSP time adjusted (ms) & $7238 \pm 3618$ & $29 \mid 4-15,726$ & $5846 \pm|83|$ & $3413-10,562$ & 0.07 \\
\hline SSP IE & $117 \pm 89$ & $36-450$ & $80 \pm 30$ & $41-157$ & $0.04 *$ \\
\hline SWM btw errors & $44 \pm 18.6$ & $7-69$ & $34.6 \pm 20.4$ & $0-72$ & $0.02 *$ \\
\hline SWM total errors & $46 \pm 19$ & $7-73$ & $36.2 \pm 21$ & $0-76$ & $0.02 *$ \\
\hline SWM strategy & $36.1 \pm 5.3$ & $23-43$ & $34.5 \pm 6.2$ & $20-46$ & 0.14 \\
\hline SWM time adjusted (ms) & $34,657 \pm 8189$ & $|8,537-48,24|$ & $29,142 \pm 7887$ & $17,944-55$ & $0.0 I^{*}$ \\
\hline SWM IE & $728 \pm 294$ & $205-1304$ & $510 \pm 252$ & $|87-||4|$ & $0.004^{* *}$ \\
\hline Digit recall & $4.6 \pm 0.8$ & $3-6$ & $4.6 \pm 0.9$ & $3-6$ & 0.21 \\
\hline Backward digit recall & $2.3 \pm 1.3$ & $1-6$ & $3.2 \pm 1.6$ & $1-6$ & $0.02 *$ \\
\hline Depression (DASS) & $6.9 \pm 3.7$ & $1-16$ & $4.9 \pm 2.9$ & $0-12$ & $0.03 *$ \\
\hline Anxiety (DASS) & $5.2 \pm 2.9$ & $1-12$ & $3.9 \pm 2.7$ & $0-10$ & 0.13 \\
\hline Stress (DASS) & $5.6 \pm 2.9$ & $1-12$ & $4.1 \pm 3.2$ & $0-12$ & 0.08 \\
\hline
\end{tabular}

Notes: Values are expressed as mean \pm SD; ${ }^{*} p<0.05$ and $* * p<0.01$ are statistically significant. For the cognitive tests, $p$-values are derived from the comparison using generalized linear models while controlling for age, sex, education.

Abbreviations: MMSE, Mini-Mental State Examination; MOT, Motor Screening Test; SSP, Spatial Span Test; SWM, Spatial Working Memory; IE, inverse efficiency; DASS, Depression Anxiety Stress Scales.

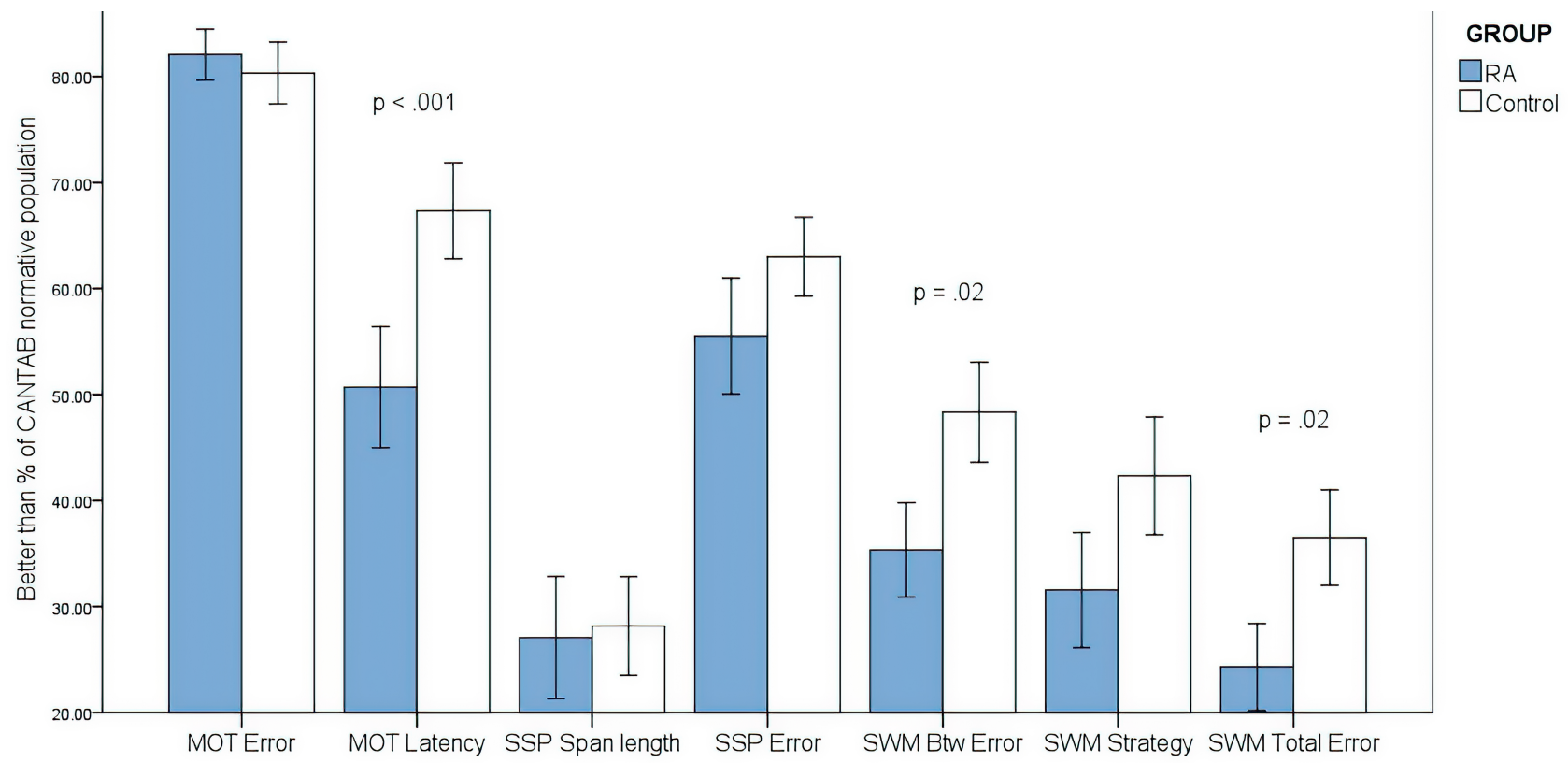

Figure I Comparison between the RA and control groups (better than \% of the age and gender-matched normative CANTAB database).

CANTAB database revealed that both samples underperformed (less than 50\%) on the SSP and the SWM measures, which suggests that cultural differences (or the fact that both samples were recruited in clinical settings) could be responsible for this pattern of deficits. Additionally, RA patients had a significantly lower verbal working memory span on the backwards digit recall test, which confirms similar previous findings in this population. ${ }^{6}$

The fractionated pattern of results for the memory tasks can be explained by the fact that unlike working memory 
Table 3 Correlations Between Cognitive and Affective Measures and Disease-Related Parameters in the RA Group

\begin{tabular}{|c|c|c|c|c|c|c|c|}
\hline & Age (Years) & Education (Years) & Disease Duration & BMI & HAQ & DAS28-CRP & MMSE \\
\hline \multicolumn{8}{|l|}{ Cognitive } \\
\hline MOT latency & 0.14 & 0.11 & 0.31 & 0.12 & 0.18 & -0.07 & 0.04 \\
\hline МOT error & 0.08 & -0.13 & -0.08 & 0.03 & -0.00 & 0.15 & 0.10 \\
\hline SSP span length & 0.00 & 0.24 & 0.00 & $-0.37^{*}$ & -0.30 & -0.28 & 0.24 \\
\hline SSP total errors & 0.26 & 0.33 & -0.06 & -0.26 & -0.12 & -0.23 & 0.06 \\
\hline SWM between errors & $0.45^{*}$ & 0.00 & 0.13 & $0.44^{*}$ & 0.07 & 0.14 & -0.24 \\
\hline SSP IE & 0.19 & -0.05 & 0.10 & -0.13 & -0.10 & -0.10 & 0.04 \\
\hline SWM total errors & $0.40 *$ & -0.02 & 0.08 & $0.48 * *$ & 0.11 & 0.20 & -0.29 \\
\hline SWM strategy & $0.39 *$ & -0.05 & 0.25 & 0.08 & -0.21 & 0.08 & 0.15 \\
\hline SWM IE & $0.50 * *$ & -0.24 & $0.36 * *$ & $0.42 * *$ & 0.04 & 0.18 & -0.12 \\
\hline Forward digit span & -0.01 & 0.16 & 0.07 & -0.25 & -0.24 & -0.07 & $0.4 I^{*}$ \\
\hline Backward digit span & $-0.47^{*}$ & 0.07 & -0.11 & -0.19 & -0.04 & -0.22 & -0.16 \\
\hline \multicolumn{8}{|l|}{ Affective } \\
\hline DASS - Depression & 0.13 & $-0.48^{*}$ & -0.09 & -0.07 & 0.41 & $0.43^{*}$ & -0.17 \\
\hline DASS - Anxiety & -0.04 & $-0.63 * *$ & -0.04 & 0.03 & $0.50 *$ & 0.39 & -0.34 \\
\hline DASS - Stress & 0.02 & $-0.54 * *$ & -0.18 & -0.05 & 0.40 & 0.36 & $-0.44^{*}$ \\
\hline
\end{tabular}

Note: ${ }^{*} \mathrm{p}<0.05$ and $* * \mathrm{p}<0.01$ are statistically significant.

Abbreviations: MOT, Motor Screening Test; SSP, Spatial Span Test; SWM, Spatial Working Memory; IE, inverse efficiency; DASS, Depression Anxiety Stress Scales; BMI, body mass index; HAQ, Health Assessment Questionnaire; DAS28-CRP, Disease Activity Score 28 Joints Count-CRP; MMSE, Mini-Mental State Examination.

demanding task, storage capacity could be less sensitive to pain modulated executive deficits in RA patients, and also less influenced by their affective problems. ${ }^{7}$ Previous studies also documented low range of impairments in RA participants at the simple storage level, in terms of visuospatial ${ }^{13}$ and verbal ${ }^{24}$ performance, while pinpointing to larger or similar deficits in more executive demanding working memory measures. ${ }^{13}$ However, these studies were not controlling for baseline differences in motor reaction speed, which is a net advantage of the current study. Our study suggests that in RA patients, for the low demanding short-term storage tasks the burden of the cognitive impairment is more taxing in terms of task efficiency than task accuracy, while for the more executive demanding working memory tests both execution accuracy and efficiency are negatively affected.

As expected, the simple reaction time task (MOT) revealed a longer time needed for RA patients to correctly react to the stimuli, confirming similar findings of sensorimotor deficits on computerized testing. ${ }^{16}$ RA specific peripheral inflammation leads to increased circulating cytokines, which have been associated with perturbed neural reactivity within the substantia nigra and with performance slowing. ${ }^{32}$

Looking at interrelations with anthropometric measures, we found increased BMI to be correlated with worse performance on both visuospatial memory, but not on verbal memory tasks (a relationship not confirmed in the control sample). The relationship between adiposity and cognition seems complex and dynamic in time, with longitudinal studies in the general population showing both protective $\mathrm{e}^{33}$ and detrimental ${ }^{34}$ roles of BMI on brain functioning. Our study reported no relationship between cognitive performance and disease activity, in line with a recent study by Said et al. ${ }^{35}$ However, we must take into consideration that RA patients in both these studies had moderate cumulative disease activity and mild/moderate functional impairment. Education was another parameter of the RA group which was not associated with cognitive impairment (although in the control sample it was negatively associated with motor slowing and with higher verbal working memory). At odds with our findings, another recent study performed on 464 RA patients reported both high disease activity and low education to be associated with cognitive impairment. ${ }^{36}$ The aforementioned study found no relationship between cognitive performance and functional status, in accordance with our data. These heterogeneous results may be explained by various cognitive assessment tools that were used in such studies.

In accordance with a meta-analysis that included 72 RA studies, ${ }^{37}$ we found the RA group to have experienced 
more depression than controls, which is a variable also linked to psychomotor slowing. ${ }^{38}$ Furthermore, we found education to be inversely correlated with all affective measures in the RA group, which suggests that education could be an important buffer for the impact of this condition on the emotional wellbeing of the participants. However, the results of our exploratory correlational analysis need to be treated with caution, and confirmed on larger prospective samples, while also adjusting for the multiple correlations being conducted.

Among other study limitations, due to the crosssectional design, no cause-effect conclusions can be directly drawn from our research, in order to highlight the actual path towards cognitive impairment. Secondly, with a relatively small sample size, we could not divide the RA patients into subgroups of gender, anthropometric measurements or other risk factors and analyze how they might have influenced the cognitive outcomes, although the correlations with these parameters were analyzed. Thirdly, duration of testing was limited so we only employed a narrow battery of cognitive tests, which omits relevant aspects of memory documented to be affected in RA patients, such as episodic memory or longer-term recall. ${ }^{13,23}$ However, all of our measures converged in addressing different facets of short-term and working memory, themselves affected by other (not measured) variables such as verbal fluency, spatial processing or general intelligence. Other authors ${ }^{39}$ recommend the analysis of differences between specific CANTAB tests as opposed to the use of aggregate, latent variables (eg, "memory" or "executive functions"), which are still commonly employed in the few studies administering more indepth cognitive tasks to RA patients. ${ }^{6}$ Finally, besides the advantages of computerized testing described above, our study shares the limitations of these studies, such as the lack of familiarity with computerized interfaces which can be intimidating for elderly participants, ${ }^{40}$ potentially obscuring differential task engagement and other qualitative indicators of performance which are not objectively recorded. ${ }^{41}$

Despite these limitations, to the best of our knowledge, this is the first study that uses a touchscreen technology in RA patients, a procedure which minimizes motor demands, and is recorded with minimal interference from the researcher during data acquisition. While the known hand deformities and motor deficits specific to RA could still play a role, their influence is minimized compared to classical executive functions assessment procedures, which rely on drawing or writing responses. ${ }^{36}$ Moreover, the IE measure which showed the greatest differences between the samples was specifically designed to reveal more purely cognitive speed-accuracy tradeoffs, only visible through such comprehensive computerized assessment. Such tradeoffs affect patients' everyday activities, since they need to employ more time and resources to achieve comparable levels of accuracy, resulting in subjective complaints of poorer concentration, word-finding difficulties and memory deficits. ${ }^{42}$

\section{Conclusion}

The findings of this study emphasize the importance of performing objective tests in order to detect subtle signs of cognitive impairment and to adopt a timely therapeutic approach. Such an intervention could include visual working memory training strategies ${ }^{43}$ based on similar methods to the ones we used for identifying the deficits. A pioneering program of memory training (eg, training both memory capacity with tasks similar to our assessment and memory strategies) has been documented to ameliorate cognitive performance and at the same time reduce inflammation in aging participants, being associated with lower peripheral levels of the $\mathrm{C}$ and pro-inflammatory cytokines. ${ }^{44}$ This opens up new avenues for using such brief testing batteries in the assessment and design of subsequent memory training endeavors which can improve quality of life and actively minimize the behavioral sequence of chronic inflammation characterizing this condition.

\section{Abbreviations}

RA, rheumatoid arthritis; fMRI, functional magnetic resonance imaging; MMSE, Mini-Mental State Examination; CANTAB, Cambridge Neuropsychological Test Automated Battery; AWMA, Automated Working Memory Assessment; ACR, American College of Rheumatology; EULAR, European League against Rheumatism; DAS28-CRP, Disease Activity Score-28 using CRP; HAQ, Health Assessment Questionnaire; CRP, C-reactive protein; RF, rheumatoid factor; anti$\mathrm{CCP}$, anti-cyclic citrullinated peptide antibodies; ELISA, enzyme-linked immunosorbent assay; MOT, Motor Screening Test; SSP, Spatial Span Test; SWM, Spatial Working Memory; IE, inverse efficiency.

\section{Ethics Approval}

This study was performed in line with the principles of the Declaration of Helsinki. Approval was granted by the 
Ethics Committee of the "Iuliu Hatieganu" University of Medicine and Pharmacy (Date 02.06.2015/No. 319).

\section{Consent for Publication}

Informed consent from individuals to publish their data in an anonymized form was obtained.

\section{Authors' Information}

Cristian Vasile Petra MD is an Internist and currently a $\mathrm{PhD}$ Candidate at the Department of Rheumatology, "Iuliu Hatieganu" University of Medicine and Pharmacy, Cluj-Napoca, Romania.

Monica Buta is a PhD-candidate at the Department of Psychology, Babeș-Bolyai University, trained to apply neuropsychological tests batteries such as CANTAB and AWMA.

Laura Visu-Petra is an Associate Professor at BabeşBolyai University and has extensive experience and relevant publications in the field of neuropsychological assessment for various clinical conditions (Down's syndrome, epilepsy).

Oana Benga is a Professor at the Department of Psychology, Babeș-Bolyai University, with expertise and publications in the neuropsychological assessment of typical and atypical development.

Maria-Magdalena Tămaş $\mathrm{MD}, \quad \mathrm{PhD}, \quad$ is a Rheumatologist, Lecturer at the Department of Rheumatology, "Iuliu Hatieganu” University of Medicine and Pharmacy, Cluj-Napoca, Romania.

Simona Rednic $\mathrm{MD}, \mathrm{PhD}$, is a Rheumatologist and Internal Medicine doctor, Professor at the Department of Rheumatology, "Iuliu Hatieganu” University of Medicine and Pharmacy, Cluj-Napoca, Romania, President-Elect of the Romanian Society of Rheumatology.

\section{Data Sharing Statement}

All study data are available upon request from the authors.

\section{Acknowledgments}

The authors are grateful to M. Ilea, M. Imbuzan, A. Camuescu, and V. Cacuci who helped with the data collection process.

\section{Author Contributions}

All authors made a significant contribution to the work reported, whether that is in the conception, study design, execution, acquisition of data, analysis and interpretation, or in all these areas; took part in drafting, revising or critically reviewing the article; gave final approval of the version to be published; have agreed on the journal to which the article has been submitted; and agree to be accountable for all aspects of the work.

\section{Funding}

A CEEX -ET 54 (2006-2008) grant awarded by the Romanian National University Research Council to coauthor Prof. Oana Benga, Department of Psychology, Babeș-Bolyai University, facilitated our access to the CANTABeclipseTM 3.0.0.

\section{Disclosure}

The authors report no conflicts of interest in this work.

\section{References}

1. Cutolo M, Kitas GD, van Riel PLCM. Burden of disease in treated rheumatoid arthritis patients: going beyond the joint. Semin Arthritis Rheum. 2014;43(4):479-488. doi:10.1016/j.semarthrit.2013.08.004

2. Bartolini M, Candela M, Brugni M, et al. Are behaviour and motor performances of rheumatoid arthritis patients influenced by subclinical cognitive impairments? A clinical and neuroimaging study. Clin Exp Rheumatol. 2002;20(4):491-497.

3. Shin SY, Katz P, Wallhagen M, Julian L. Cognitive impairment in persons with rheumatoid arthritis. Arthritis Care Res (Hoboken). 2012;64(8):1144-1150. doi:10.1002/acr.21683

4. Wallin K, Solomon A, Kreholt I, Tuomilehto J, Soininen H, Walin K. Midlife rheumatoid arthritis increases the risk of cognitive impairment two decades later: A population-based study. J Alzheimer's Dis. 2012;31(3):669-676. doi:10.3233/JAD-2012-111736

5. Yoon BY, Lee J, Shin SY. Discrepancy between subjective and objective measures of cognitive impairment in patients with rheumatoid arthritis. Rheumatol Int. 2017;37(10):1635-1641. doi:10.1007/ s00296-017-3806-2

6. Meade T, Manolios N, Cumming SR, Conaghan PG, Katz P. Cognitive Impairment in Rheumatoid Arthritis: A Systematic Review. Arthritis Care Res (Hoboken). 2018;70(1):39-52. doi:10.1002/acr.23243

7. Abeare CA, Cohen JL, Axelrod BN, Leisen JCC, Mosley-Williams A, Lumley MA. Pain, executive functioning, and affect in patients with rheumatoid arthritis. Clin J Pain. 2010;26(8):683-689.

8. Covic T, Cumming SR, Pallant JF, et al. Depression and anxiety in patients with rheumatoid arthritis: prevalence rates based on a comparison of the Depression, Anxiety and Stress Scale (DASS) and the hospital, Anxiety and Depression Scale (HADS). BMC Psychiatry. 2012;12(1):6. doi:10.1186/1471-244X-12-6

9. Kaplan C, Minc A, Basu N, Schrepf A. Inflammation and the central nervous system in inflammatory rheumatic disease. Curr Rheumatol Rep. 2019;21(12):67. doi:10.1007/s11926-019-0870-5

10. Sağ S, Serdar M, Ibrahim S, et al. Central nervous system involvement in rheumatoid arthritis: possible role of chronic inflammation and TNF blocker therapy. Acta Neurol Belg. 2017;120(1):25-31. doi:10.1007/s13760-017-0879-3

11. Folstein MF, Folstein SE, McHugh PR. "Mini-mental state". A practical method for grading the cognitive state of patients for the clinician. J Psychiatr Res. 1975;12(3):189-198. doi:10.1016/ 0022-3956(75)90026-6 
12. Vitturi BK, Nascimento BAC, Alves BR, de Campos FSC, Torigoe DY. Cognitive impairment in patients with rheumatoid arthritis. $J$ Clin Neurosci. 2019;69:81-87. doi:10.1016/j. jocn.2019.08.027

13. Shin SY, Katz P, Julian L. Relationship between perceived cognitive dysfunction and objective neuropsychological performance in persons with rheumatoid arthritis. Arthritis Care Res (Hoboken). 2013;65(3):481-486. doi:10.1002/acr.21814

14. Kim SH, Kim SH, Kim SK, Nam EJ, Han SW, Lee SJ. Spatial versus verbal memory impairments in patients with fibromyalgia. Rheumatol Int. 2012;32(5):1135-1142. doi:10.1007/s00296-010-1762-1

15. Barraclough M, McKie S, Parker B, et al. Altered cognitive function in systemic lupus erythematosus and associations with inflammation and functional and structural brain changes. Ann Rheum Dis. 2019;78 (7):934-940. doi:10.1136/annrheumdis-2018-214677

16. Hanly JG, Omisade A, Su L, Farewell V, Fisk JD. Assessment of cognitive function in systemic lupus erythematosus, rheumatoid arthritis, and multiple sclerosis by computerized neuropsychological tests. Arthritis Rheumatism. 2010;62(5):1478-1486.

17. CANTABeclipse Test Administration Guide. Manual Version 3.0.0. Cambridge Cognition Limited; 2006.

18. Alloway TP. Automated Working Memory Assessment (AWMA). London: Harcourt Assessment; 2007.

19. Giedraitiene N, Kaubrys G. Distinctive pattern of cognitive disorders during multiple sclerosis relapse and recovery based on computerized CANTAB tests. Front Neurol. 2019;10:(JUN):1-10. doi:10.3389/ fneur.2019.00572

20. Visu-Petra L, Benga O, Tincas I, Miclea M. Visual-spatial processing in children and adolescents with Down's syndrome: A computerized assessment of memory skills. J Intellect Disabil Res. 2007;51 (12):942-952. doi:10.1111/j.1365-2788.2007.01002.x

21. Bogaczewicz A, Sobow T, Kowalski J, Ząbek J, Woźniacka A, Bogaczewicz J. Cambridge neuropsychological Test Automated Battery in assessment of cognitive parameters in patients with systemic lupus erythematosus in relation to autoantibody profile. Reumatologia. 2015;53(3):131-138. doi:10.5114/reum.2015.53134

22. Julian LJ, Yazdany J, Trupin L, Criswell LA, Yelin E, Katz PP. Validity of brief screening tools for cognitive impairment in rheumatoid arthritis and systemic lupus erythematosus. Arthritis Care Res (Hoboken). 2012;64(3):448-454. doi:10.1002/acr.21566

23. Medina LD, Hirshberg L, Taylor MJ, Gilbert PE, Heaton RK. Rates of Neuropsychological Dysfunction in Fibromyalgia and Rheumatoid Arthritis: an Automated Clinical Rating Approach. J Clin Rheumatol. 2019;25(6):252-257. doi:10.1097/RHU.0000000000000837

24. Petersen LE, Baptista TSA, Molina JK, et al. Cognitive impairment in rheumatoid arthritis: role of lymphocyte subsets, cytokines and neurotrophic factors. Clin Rheum. 2018;37(5):1171-1181. doi:10.1007/s10067-018-3990-9

25. Aletaha D, Neogi T, Silman AJ, et al. 2010 Rheumatoid arthritis classification criteria: an American College of Rheumatology/ European League Against Rheumatism collaborative initiative. Arthritis Rheum. 2010;62(9):2569-2581. doi:10.1002/art.27584

26. Prevoo MLL, Van'T Hof MA, Kuper HH, Van Leeuwen MA, Van De Putte LBA, Van Riel PLCM. Modified disease activity scores that include twenty-eight-joint counts. Development and validation in a prospective longitudinal study of patients with rheumatoid arthritis. Arthritis Rheum. 1995;38(1):44-48. doi:10.1002/ art.1780380107

27. Fries JF, Spitz P, Kraines RG, Holman HR. Measurement of patient outcome in arthritis. Arthritis Rheum. 1980;23(2):137-145. doi:10.1002/art.1780230202

28. Munteanu C-E, Iliescu D, Livinti R. MMSE-2: Mini-Mental State Examination, 2nd Edition. Manual De Utilizare a Testului. Sinapsis, Cluj-Napoca; 2013.
29. Visu-Petra L, Cheie L, Benga O, Packiam Alloway T. Effects of anxiety on memory storage and updating in young children. Int J Behav Dev. 2011;35(1):38-47. doi:10.1177/0165025410368945

30. Lovibond SH, Lovibond LP. Manual for the Depression Anxiety Stress Scales. Sydney: Psychology Foundation; 1995.

31. Perte A, Albu M. DASS Manual Pentru Scalele De Depresie, Anxietate Si Stres. Adaptarea Si Standardizarea Pe Populatia Din Romania. Cluj-Napoca: ASCR; 2011.

32. Brydon L, Harrison NA, Walker C, Steptoe A, Critchley HD. Peripheral inflammation is associated with altered substantia nigra activity and psychomotor slowing in humans. Biol Psychiatry. 2008;63(11):1022-1029. doi:10.1016/j.biopsych.2007.12.007

33. Alhurani RE, Vassilaki M, Aakre JA, et al. Decline in weight and incident mild cognitive impairment Mayo Clinic study of aging. JAMA Neurol. 2016;73(4):439-446. doi:10.1001/jamaneurol.2015.4756

34. Gallucci M, Mazzuco S, Ongaro F, et al. Body mass index, lifestyles, physical performance and cognitive decline: the "Treviso Longeva (TRELONG)" study. J Nutr Heal Aging. 2013;17(4):378-384. doi:10.1007/s12603-012-0397-1

35. Said FA, Betoni TB, Magalhaes V, Nisihara R, Skare TL. Rheumatoid arthritis and cognition dysfunction: lack of association with cumulative glucocorticoid use. Immunopharmacol Immunotoxicol. 2019;41(6):565-567. doi:10.1080/ 08923973.2019.1679170

36. Katchamart W, Narongroeknawin P, Phutthinart N, Srinonprasert V, Muangpaisan W, Chaiamnauy S. Disease activity is associated with cognitive impairment in patients with rheumatoid arthritis. Clin Rheumatol. 2019;38(7):1851-1856. doi:10.1007/s10067-019-04488-3

37. Matcham F, Rayner L, Steer S, Hotopf M. The prevalence of depression in rheumatoid arthritis: A systematic review and meta-analysis. Rheumatol. 2013;52(12):2136-2148. doi:10.1093/rheumatology/ ket169

38. White DA, Myerson J, Hale S. How cognitive is psychomotor slowing in depression? Evidence from a meta-analysis. Aging Neuropsychol Cogn. 1997;4(3):166-174. doi:10.1080/13825589708256645

39. Haring L, Mõttus R, Koch K, Trei M, Maron E. Maron E Factorial validity, measurement equivalence and cognitive performance of the Cambridge Neuropsychological Test Automated Battery (CANTAB) between patients with first-episode psychosis and healthy volunteers. Psychol Med. 2015;45(9):1919-1929. doi:10.1017/ S0033291714003018

40. Zygouris S, Tsolaki M. Computerized cognitive testing for older adults: A review. Am J Alzheimers Dis Other Demen. 2015;30 (1):13-28. doi:10.1177/1533317514522852

41. Wild K, Howieson D, Webbe F, Seelye A, Kaye J. Status of computerized cognitive testing in aging: A systematic review. Alzheimer's Dement. 2008;4(6):428-437. doi:10.1016/j.jalz.2008.07.003

42. Shadick NA, Katz P, Iannaccone CI, et al. The impact of exercise, lifestyle, and clinical factors on perceived cognitive function in patients with rheumatoid arthritis: results from a prospective cohort study. ACR Open Rheumatol. 2019;1(10):620-626. doi:10.1002/ acr2.11088

43. Bi T, Wang X, Kou H, Qiu J. The effect of visual working memory training could transfer across stimuli. Psychol Res Behav Manag. 2020;13:55-66. doi:10.2147/PRBM.S240526

44. Pesce M, Tatangelo R, La Fratta I, et al. Memory training program decreases the circulating level of cortisol and pro-inflammatory cytokines in healthy older adults. Front Mol Neurosci. 2017;10: (July):1-17. doi:10.3389/fnmol.2017.00233 


\section{Publish your work in this journal}

Psychology Research and Behavior Management is an international, peer-reviewed, open access journal focusing on the science of psychology and its application in behavior management to develop improved outcomes in the clinical, educational, sports and business arenas. Specific topics covered in the journal include: Neuroscience, memory and decision making; Behavior modification and management; Clinical

applications; Business and sports performance management; Social and developmental studies; Animal studies. The manuscript management system is completely online and includes a very quick and fair peer-review system, which is all easy to use. Visit http://www. dovepress.com/testimonials.php to read real quotes from published authors. 